\title{
Estructura factorial de la escala de satisfacción con la vida y validez conver- gente con la escala de calidad de vida en preparatorianos mexicanos
}

\section{Factor structure of satisfaction with life scale and convergent validity with the quality of life scale in high school Mexican students}

\author{
Martell Muñoz, Juan ; García-Cadena, Cirilo Humberto²; Daniel-González, Leopoldo ${ }^{2,}$; Sán- \\ chez Miranda Patricia ${ }^{1}$ y Mendoza Martínez, Abraham²
}

\begin{abstract}
Resumen:
El propósito de este estudio fue calcular tanto la validez de constructo como la validez convergente de la escala de Satisfacción con la Vida (SWL), de Diener, Emmons, Larsen y Griffin (1985), en 428 estudiantes de bachillerato. Se usaron análisis factorial exploratorio y confirmatorio. Diener et al. (1985) diseñan una escala que estandariza la medida de la satisfacción con la vida, de manera válida y confiable. Se encuentra que las $\lambda$ 's de los ítems tienen un recorrido desde .58 hasta .88 . El coeficiente de consistencia interna alfa de Cronbach es de .85 , con un intervalo de confianza del $95 \%$ (de .826 a .871). El coeficiente de correlación de Pearson entre la SWLS y la escala de Calidad de Vida es de $\mathrm{r}=.395 ; \mathrm{p}<.01 ; \mathrm{IC} 95 \%$ : .293, .486. Hay coincidencia entre los hallazgos arrojados por ambos tipos de análisis factorial. El modelo de medición se corresponde con el creado por Diener et al. (1985): los cinco ítems muestran validez y confiabilidad, al igual que lo reportado por otros estudios en diferentes contextos culturales.
\end{abstract}

Palabras Clave: satisfacción con la vida, análisis factorial, validez convergente, estudiantes mexicanos, bachillerato.

\begin{abstract}
:
The purpose of this study was to calculate the construct and convergent validity of the Satisfaction with Life Scale (SWL), Diener, Emmons, Larsen and Griffin (1985) in 428 high school students. Exploratory and confirmatory factor analyses were used. Diener et al. (1985) designed a scale that standardize the measure of satisfaction with life, in a valid and reliable way. Results show that $\lambda$ 's of the items have a spread from .58 to .88 . The internal consistency coefficient Cronbach's alpha is .85 , with a confidence interval of $95 \%$ (.826- . 871). Pearson coefficient correlation between SWLS and Quality Life Scale is $\mathrm{r}=.395 ; \mathrm{p}<.01$; IC95\%: .293, .486. There is agreement between the findings thrown by both types of factor analysis. The measure of model corresponds to the created for Diener et al. (1985): the five items show validity and reliability, as reported by other studies in different cultural contexts.
\end{abstract}

Keywords: satisfaction with life, factor analysis, convergent validity, Mexican students, high school.

\footnotetext{
${ }^{1}$ Universidad Autónoma de Zacatecas, México.

${ }^{2}$ Universidad Autónoma de Nuevo León, México.

*Correspondencia: leopoldo.danielgn@uanl.edu.mx
} 
México se considera un país de jóvenes, de acuerdo con los datos proporcionados por la Encuesta Intercensal, en el 2015 existían $25.7 \%$ de jóvenes con edades entre los 15 a 29 años. De los cuales $35.1 \%$ son adolescentes con edades de 15 a 19 años (Instituto Nacional de Estadística y Geografía [INEGI], 2015). Esto resulta significativo, ya que esta es una de las etapas de la vida que se ubica en transición entre la infancia y la adultez (Lozano, 2014), caracterizada por presentar una serie de cambios a nivel físico, ocasionados por el súbito aumento en las hormonas. Además, acompañada por cambios psicológicos, con avances significativos al desarrollar un pensamiento más abstracto. Lo cual le permite ser más critico, tendencias a discutir, pero al mismo tiempo carece de suficientes estrategias para realizar una buena elección entre diversas actividades, aun y cuando sean sencillas (Papalia, Wendkos, y Duskin, 2001).

Asimismo, son autoconscientes de su persona como de las demás personas. Su lenguaje se ve enriquecido al comprender conceptos abstractos como justicia, amor, libertad. Por otro lado, su evolución psicosocial permite la consolidación de su identidad, que junto con la educación accede a la elaboración de un sentido del yo. En este aspecto, también resulta un periodo critico, ya que es cuando se presentan problemas delictivos, enfermedades de transmisión sexual, drogadicción, entre otros (Papalia, et al., 2009)

Ante este panorama de la adolescencia, la Organización de las Naciones Unidas (ONU, 2010), preocupada por mejorar el bienestar y la calidad de vida de los jóvenes, establece el Programa de Acción Mundial para los Jóvenes (PAMJ), donde se especifican una serie de estrategias y programas que coadyuven en el logro de las metas. Con la finalidad de lograr conocer el bienestar de los adolescentes resulta esencial contar con las herramientas que nos permitan detectarlo, por ello es importante identificar las características psicométricas de los instrumentos usados para medir el bienestar subjetivo, dentro de la población mexicana y en particular de los adolescentes.

El concepto de bienestar es utilizado en diferentes disciplinas como en sociología, salud, economía y en psicología, entre otras. En psicología, siguiendo una tradición filosófica, se evalúa con conceptos de felicidad, optimismo, calidad de vida y satisfacción, abriendo el surgimiento de dos líneas de investigación. Por un lado, el bienestar subjetivo y por el otro, el bienestar psicológico. En el primero se incluyen los aspectos afectivos, emocionales y cognitivos de las personas o grupos, sus investigaciones incluyen principalmente los constructos de satisfacción con la vida, la felicidad y calidad de vida. Mientras que el segundo trabaja con el funcionamiento positivo del individuo. Se compone de múltiples dimensiones (autoaceptación, autonomía, crecimiento personal, propósito en la vida, relaciones positivas con otros y dominio del entorno) (Rodríguez y Quiñones, 2012).

En la presente investigación se trabajó con los constructos de satisfacción con la vida y calidad de vida, los cuales son componentes importantes del bienestar subjetivo. Asimismo, se consideró la felicidad como elemento de relevancia del bienestar.

\section{Satisfacción con la vida}

La satisfacción con la vida implica disponer de varios gratificantes que construyan o permitan construir una significación y simbolización de lo que se entiende como estar satisfecho con lo que se tiene y con lo que se vive (en dicha definición de manera implícita se ponen de manifiestos algunos componentes 
de la felicidad). A finales de los años sesenta Wilson (1967), después del análisis de diferentes factores correlacionados con la felicidad llegó a la conclusión de que una persona feliz es una persona joven, con buenos ingresos, alegre, extrovertida, con aspiraciones modestas, alta autoestima, libre de preocupaciones, con una moralidad laboral conservadora, inteligente, etc. A pesar del sesgo ideológico de la abstracción de Wilson, se obtiene un interés primario en la felicidad para las Ciencias Sociales. Aquí cabe mencionar los estudios señeros de Jahoda (1958) como influencia y catapulta de los estudios de la Psicología Positiva. Por su parte, Diener, Horowitz y Emmons (1985), estudian la felicidad de los más ricos de la lista Forbes y personas de bajo y medio ingreso, encontrando que el dinero no tiene relación directa con la felicidad. Éste interesante hallazgo demuestra que la satisfacción con la vida y la felicidad no se relacionan con el dinero. Sin embargo, el dinero sí dota de ciertas competencias para hacerle frente a ciertas circunstancias de la vida.

Más recientemente y en la misma dirección, Herian, Tay, Hamm y Diener (2014) demuestran que la salud, e implícitamente la satisfacción con la vida, se encuentran más ligados al capital social ya que se le dota a la población de una serie de competencias formales e informales para la vida en democracia y para la vida en general. A finales de los años ochenta y principios de los noventa del siglo pasado empieza a emerger una serie de disciplinas y subdisciplinas dentro de la psicología, las cuales pretendían dar respuesta del por qué algunas personas resultaban más felices que otras. Estudios acerca de temas emergentes como la resiliencia (Werner \& Smith, 1982), satisfacción con la vida (Diener, Horowitz, \& Emmons, 1985), bienestar subjetivo (Diener, 1984), bienestar psi- cológico (Ryff \& Singer, 1998), bienestar social (Keyes, 1998), calidad de vida, etc., se han convertido en objetos de estudio de lo humano desde una perspectiva más psicológica. Esta psicología y sus subdisciplinas han transformado a lo humano en un campo de redescubrimiento y de recomposición, así como la reconceptualización dentro de la propia disciplina, en la búsqueda de las potencialidades humanas.

Diener en 1984 hace una revisión de la obra de Wilson, trabajando en la realización de una sola dimensión de los múltiples factores contemplados por Wilson. En este punto se resuelve una encrucijada entre lo que los antiguos consideraban era la felicidad, al mismo tiempo se constituye para la ciencia un constructo más manejable, así como reproducible: la satisfacción con la vida. Desde las primeras líneas de su artículo, Myers y Diener (1995) mencionan que la Psicología como disciplina ha gastado ríos de tinta para hablar de lo negativo de la vida humana: ansiedad, sufrimiento, depresión, y muy pocas, para el gozo y disfrute de la misma. Además, señalan que el estudio de la satisfacción con la vida no es una sustitución de los intereses investigativos de la psicología, sino un extraordinario complemento. Asimismo, se ha relacionado con una amplia variedad de campos como por ejemplo los economistas lo han asociado casi siempre con la capacidad de conseguir los bienes y satisfactores materiales que, sin embargo, no son los más preciados para conseguir la felicidad y/o una adaptación más o menos eficaz a una sociedad de manera plena (Myers \& Diener, 1995). Del mismo modo, Zagórski, Kelley y Evans (2010), reportan que en parte el bienestar, la felicidad y la satisfacción no están ligadas con la abundancia o la riqueza material; sin embargo, los países pobres, corruptos y con carencias en los servi- 
cios públicos, son más propensos a encontrarse menos satisfechos con la vida, a diferencia de países donde los pobres tienen acceso a la educación, salud y cuentan con una infraestructura que corresponde a sus necesidades, informan ser más felices aun y cuando en su país o su región sean considerados pobres. Fischer y Boer (2011), en un meta-análisis, también manifiestan que existe una relación entre satisfacción con la vida y la riqueza material; sin embargo, ésta es relativa a otros elementos de la vida social y con sus respectivos gradientes.

En el campo educativo se ha encontrado que la satisfacción con la vida se relaciona con el logro académico (Abu-Bakar, 2012) y con la satisfacción en la vida escolar, así como con el establecimiento y mantenimiento de relaciones armónicas en la escuela y con el resto de la vida (Lee, Lee, \& Rojewski, 2012; You et al., 2008). Los reportes con estudiantes jóvenes, señalan que las personas satisfechas con la vida no sólo muestran satisfacción con la vida escolar, sino también con otros rubros de la vida en general: menor riesgo de consumo de drogas, relaciones sociales gratificantes, menos estrés (Escobar, Blanca, Fernández-Baena, y Trianes, 2011; Proctor, Linley, \& Maltby, 2009.) A su vez Sun y Shek (2012) indican que son menos propensos a manifestar conductas disruptivas en la escuela y en otros contextos de la sociedad. En Perú se encontró que los adolescentes con edades entre los 14 a 17 años que presentaban pensamientos rumiativos correlacionan negativamente con la satisfacción con la vida (Villa, 2015).

En el campo de la salud física, como es el estudio entre la satisfacción con la vida y enfermedades crónicas, se encontró que está en función del tipo de padecimiento. Entre más difícil sea la condición para cubrir las necesidades básicas o se vean amenazadas su supervivencia su satisfacción será menor (Sánchez, García, Valverde, y Pérez, 2014). La felicidad (satisfacción con la vida) además de estar relacionada con un envejecimiento más sano, también se relaciona como medio preventivo en la salud física y con la salud mental (Veenhoven, 2008; Yiengprugsawan, Somboonsook, Sam-ang, \& Sleigh, 2012).

Como ya se mencionó la satisfacción con la vida tiene relación con aspectos sociodemográficos, culturales y económicos, tales como la edad, sexo, estrato económico, región, religión y esto hace pensar en una conceptualización multifactorial y multidimensional (Gwozd \& Sousa-Poza, 2009; Martínez, González-Arratia, van Barneveld y Domínguez, 2012; Sun, Chen, Johannesson, Kind, \& Burström, 2015). En este sentido, es posible encontrar que los adolescentes que se encuentran apegados al lugar, también poseen una correlación positiva con el bienestar, específicamente con la satisfacción con la vida (Magalhães \& Calherios, 2015). Pero también es importante considerar las condiciones de una nación como: la paz, honestidad, justicia y mejores condiciones de vida, lo cual puede llevar a la percepción de satisfacción que las personas desean (Martínez, 2006).

De acuerdo a literatura consultada, dentro de la psicología positiva, la satisfacción con la vida y la felicidad se manejan como sinónimos dado que ambas son componentes del bienestar subjetivo, sin embargo, también es posible establecer diferencia entre las dos. Aun y cuando la felicidad se ha considerado como un constructo complejo, por poseer una amplia variedad de definiciones que se le han adjudicado, Alarcón (2006), en un esfuerzo por construir un instrumento capaz de medirla en un contexto cultural del habla hispana sudamericana, retoma las ideas 
filosóficas griegas e investigaciones acerca del bienestar subjetivo y la felicidad. La define como un estado afectivo con una valoración positiva, donde se incluye una satisfacción por la posesión de un bien anhelado, este bien puede ser de diversos tipos: materiales, religiosos, psicológicos, etc. Asimismo, esta concepción sugiere una temporalidad.

Mientras que la satisfacción con la vida se considera que corresponde al componente cognitivo del bienestar subjetivo, donde el individuo realiza una valoración general o global de su vida, también puede realizarla en aspectos específicos. Dentro de dicha valoración realiza una comparación entre su vida actual y lo que espera, esto es, realiza una reflexión y valoración entre las aspiraciones y sus logros (Diener, Emmos, Larsen, \& Griffin, 1985; Diener, Oishi, \& Lucas, 2003).

Además, de acuerdo a Diener, el bienestar puede ser explicado por diversas teorías, una de ellas es la universalista, donde la satisfacción se alcanza gracias al logro de los objetivos o cuando se satisfacen las necesidades básicas. Y estas necesidades se ven moderadas por las emociones, el aprendizaje, la cultura, etc. (Castro, 2009).

Ryff y Singer (1998) mencionan que las causas del bienestar son iguales para todas las personas. Estas causas son: el propósito de vida, la calidad de las relaciones, el sentimiento de dominio y la autoestima. Los autores que se basan en el estudio de la autodeterminación aseguran, que ésta posee un componente innato y que deberían de manifestar en todas las sociedades. Y las diferencias que existen entre las culturas son debidas a los niveles de autonomía, significados y relaciones que se les proporcionan (Tov \& Diener, 2007).

\section{La medición de la satisfacción con la vida}

Uno de los instrumentos de autoreporte más utilizados para medir la satisfacción con la vida es la propuesta por Diener, Emmons, Larsen, y Griffin (1985). La satisfacción con la vida se diferencia del bienestar al considerar que la primera incluye los juicios sobre los procesos cognitivos y bajo esta idea se construye una escala que se focaliza en dichos procesos, dejando a un lado los aspectos afectivos positivos y negativos, aspectos que si se consideran en el bienestar (Atienza, Pons, Balaguer, y García-Merita, 2000).

Para su construcción, se inició con 48 ítems, los cuales incluían enunciados relacionados con la satisfacción con la propia vida y algunos ítems de contenido afectivo positivo y negativo. Estos últimos fueron eliminados y todos aquellos de satisfacción que cargaron con $\lambda$ 's menores a .60, quedando 10 ítems. De éstos, se retiraron otros cinco ítems que presentaban semejanzas semánticas entre ellos. Obteniendo finalmente la escala de 5 ítems. Para su validación se usó análisis factorial exploratorio, con el método de extracción de factores conocido como factorización de ejes principales (Pedhazur \& Pedhazur Schmelkin, 1991). Se obtuvieron propiedades psicométricas adecuadas (Diener et al., 1985).

Posteriormente, Lucas, Diener y Suh (1996) realizaron una serie de tres estudios con la finalidad de establecer la validez discriminante entre las mediciones de bienestar, estableciendo la diferencia entre ellas, sobre todo entre la satisfacción con la vida, afecto positivo, afecto negativo, optimismo y autoestima. En los últimos años la escala de satisfacción con la vida ha sido adaptada a diversos idiomas, así como se ha aplicado a muestras muy diversas, lo cual ha permitido corroborar la naturaleza unifactorial de la escala original, y su confiabilidad (Atienza, Pons, 
Balaguer, y García-Merita, 2000). También se han realizado investigaciones transculturales donde se confirma la invarianza factorial de la escala, así como su confiabilidad (Atienza, Balaguer, Corte-Real, \& Fonseca, 2016; Díaz, Stavraki, Blanco, \& Gandarillas, 2015; Dimitrova \& Domínguez, 2015). Por tanto, la escala se ha adaptado al castellano en muestras españolas de diferentes características: adolescentes, estudiantes universitarios, mujeres embarazadas y puérperas; en todos estos estudios se ha confirmado la estructura unifactorial de la escala, y confiabilidad, la cual oscila alrededor de .82 a .84 (Atienza et al., 2000; Cabañero et al., 2004; Garrido, Fernández, Villalba, Pérez, y Fernández-Bellido, 2010; Vázquez, Duqe, \& Hervás, 2013).

En América Latina también se ha validado la escala en países como Chile y Colombia, (Cárdenas et al., 2012; Díaz, Stavraki, Blanco \& Gandarillas, 2015). Argentina, México y Nicaragua, en personas adultas (Dimitrova \& Domínguez, 2015). En el estado de Colima, México, se aplicó en adultos de ambos géneros, se calculó la confiabilidad del instrumento, encontrando una alfa de Cronbach de .79; sin embargo, no se realizó un análisis de la validez del constructo, ya que no era el objetivo del estudio (Laca, Verdugo, y Guzmán, 2005; Martínez et al., 2012). En Michoacán, Padrós, Gutiérrez y Medina (2015), también estudiaron las propiedades psicométricas de la escala de satisfacción con la vida, a diferencia del estudio de Laca, et al. (2005), se trabajó con estudiantes de licenciatura, además de población en general. Ellos utilizaron un análisis factorial confirmatorio, detectando una estructura de un solo factor y con un $\alpha=.83$.

Por otro lado, además de la satisfacción con la vida, otro de los componentes del bienestar subjetivo, es el de calidad de vida, el cual también ha sido utilizado como sinónimo de satisfacción con la vida. No obstante, de esta comparación se ha logrado hacer una diferenciación y Palomar (1999) la ha definido como un constructo multidimensional donde se considera la evaluación de la satisfacción en diferentes áreas. Y para su evaluación toma en cuenta diferentes bloques, El primero de ellos corresponde a áreas generales de calidad de vida como la sociabilidad, la familia, bienestar económico, desarrollo personal, actividades recreativas, percepción personal. Mientras que en el segundo bloque se abordan los aspectos laborales, incluye el desarrollo personal en el trabajo y su reconocimiento individual en el mismo. El bloque tres indaga la relación de pareja y por último la relación con los hijos y el desarrollo personal. La escala demostró una consistencia interna que se encuentra entre .75 y .93 (Palomar, 1995).

Con base a la literatura teórica y empírica revisada, en este estudio se planteó el problema de si se obtendrían en alumnos mexicanos de bachillerato, de Zacatecas, México, propiedades psicométricas similares a las reportadas por las investigaciones realizadas sobre la escala de satisfacción con la vida, esto es, si al igual que en otras regiones del mundo se confirman las propiedades de la escala que pudieran apoyar la universalidad de una de las propuesta teóricas mencionadas por Diener (1984). Así, el objetivo primario del estudio fue conocer la validez de constructo y convergente, su estructura factorial subyacente y la precisión de la medida de esta tan usada escala de satisfacción con la vida, en alumnos de bachillerato. Además, se plantearon dos hipótesis, la primera de ellas: las propiedades psicométricas serán similares a las obtenidas en otras culturas, demostrando la universalidad de la escala. Y como segunda hipótesis: la escala de satisfacción con la vida 
poseerá una convergencia con la escala de calidad de vida, sin que lleguen ha ser el mismo tipo de constructo.

La presente investigación resulta de relevancia debido a la carencia de este tipo de investigaciones en México, tanto en población escolarizada como en población abierta. Y que nos permita conocer el grado de satisfacción que poseen los mexicanos, en particular los adolescentes escolarizado y, de acuerdo con tal información, buscar las estrategias para brindar mejorar sus niveles de satisfacción, en caso de ser necesario.

\section{Diseño}

\section{Método}

Se realizó un estudio de tipo instrumental. De acuerdo a Montero y León (2005) el indagar sobre las propiedades psicométricas de una prueba pertenece a esta categoría.

\section{Participantes}

Se trabajó con una muestra no probabilística de 428 estudiantes de todos los semestres del Colegio de Bachilleres de Zacatecas, México (COBAEZ), 205 (47.90\%) de sexo masculino y $223(52.10 \%)$ de sexo femenino. Debido a que no se tuvo acceso a la lista completa de estudiantes de la institución es que se tuvo que usar una muestra por conveniencia. Las edades oscilaron entre los 14 y 20 años, con una media de edad $\mathrm{M}=16.83$ y una desviación estándar $\mathrm{SD}=1.07$. No se encontraron diferencias estadísticamente significativas entre los promedios de edad de hombres $\mathrm{y}$ mujeres $(\mathrm{t}(430)=1.545, \mathrm{p}=0.001, \mathrm{~d}=.09$, IC95\%-.044 -.365).

\section{Instrumentos}

Escala de Satisfacción con la Vida. (SWLS; Diener, Emmons, Larsen, \& Griffin, 1985). Se empleó la versión española de Atienza,
Pons, Balaguer y García-Merita (2000). La escala está formada por cinco ítems y siete opciones de respuesta $(7=$ Fuertemente de acuerdo, 6= De acuerdo, 5= Ligeramente de acuerdo, 4= Ni de acuerdo ni en desacuerdo, $3=$ Ligeramente en desacuerdo, 2= En desacuerdo, 1= Fuertemente en desacuerdo) y sus puntuaciones oscilan entre 5 y 35 . La SWLS ha sido validada en universitarios mexicanos por Padrós, Gutiérrez, y Medina (2015), en este estudio la consistencia interna fue $\alpha=.832$

Escala de Calidad de Vida. (Palomar, 1995). La escala está constituida por 50 ítems y tiene tres opciones de respuesta tipo Likert (3= Poco satisfecho, 2= Regularmente satisfecho, 1= Muy satisfecho). Las propiedades psicométricas reportadas por Palomar (1995) fueron una estructura factorial de 8 factores y alfas de Cronbach de .93. Esta prueba se usó para calcular la validez convergente de la Escala de Satisfacción con la Vida. En el presente estudio, mediante el empleo del AFE por extracción de mínimos cuadrados no ponderados, se encontró la existencia de una estructura de ocho factores que explican el $55.29 \%$ de la varianza de la Escala de Calidad de Vida, con un buen indicador de consistencia interna alfa de Cronbach $\alpha=.898$.

\section{Procedimiento}

Se pidió a los alumnos respondieran la Escala de Satisfacción con la Vida de Diener et al. (1985), se les entregó el consentimiento informado apegado al código ético del Psicólogo (Sociedad Mexicana de Psicología, 2007), donde se les informaba que su participación era de forma voluntaria, que no habría sanciones en caso de negarse a participar o de dejar de hacer en cualquier momento que así lo decidieran. La información se trató de forma confidencial. 


\section{Análisis de Datos}

Se utilizaron los programas estadísticos SPSS 24 para los análisis descriptivos, correlaciones ( $r$ de Pearson), confiabilidad (alfa de Cronbach) y cálculo de normalidad mediante la prueba Kolmogorov-Smirnov, AMOS 24 para efectuar el análisis factorial confirmatorio y el R-Menú 2.4.2 para SPSS 24 para la ejecución del análisis paralelo de Horn, el cual según Courtney (2013) es un método con alta confiabilidad para determinar el número de factores. Para identificar la estructura de la escala de Satisfacción con la Vida, se realizó el análisis factorial exploratorio (AFE) y el análisis factorial confirmatorio (AFC) utilizando cuadrados mínimos libres de escala, y que según Lloret-Segura, Ferreres-Taver, Hernandez-Baeza y Tomás-Marco (2014) es el método indicado para test que no cuenta con normalidad. La bondad de ajuste del modelo se evaluó siguiendo las recomendaciones de Hancock y Mueller (2006): Chi-cuadrado $(\chi 2)$, el índice de bondad de ajuste (GFI), índice de bondad de ajuste ajustado (AGFI), índice de ajuste normalizado (NFI), y el residuo cuadrático medio estandarizado (SRMR) y el residuo cuadrático medio (RMR). Si bien la $\chi 2$ es el método más utilizado para evaluar la bondad del ajuste, tiene como limitación el ser muy sensible al tamaño muestral (Fujikoshi, 2000). Ante esto, se incorporó el cálculo de la chi-cuadrado relativo para el modelo ( $\chi 2 / \mathrm{df}$ ) (Bollen, 1998), donde valores entre dos y tres o hasta una diferencia $\leq 5$, indican un buen ajuste de modelo. En conjunto, valores GFI, AGFI y NFI > .95 así como el SRMR y RMR $<.08$ son indicadores de un adecuado ajuste entre el modelo hipotético y los datos (Hancock \& Mueller, 2006; Hu \& Bentler, 1999).

A partir de lo recomendado por Fornell y Larcker (1981) se obtuvo evidencia in- terna de validez convergente mediante el cálculo de la varianza promedio extraída del factor (AVE; Average Variance Extracted), donde valores $>.50$ son considerados satisfactorios y además se estimó el índice de confiabilidad compuesta (CR; Composite Reliability), el cuál para ser aceptable debe ser mayor a .60 y recomendado mayor a .70 . Asimismo, la evidencia de validez convergente se estimó a través del coeficiente de correlación de Pearson (r) entre la puntuación de la SWLS y otra medida que está teóricamente relacionada con la Satisfacción con la Vida como lo es la escala de Calidad de Vida (Palomar, 1995). Se calculó el tamaño del efecto (TE) de las correlaciones $(\mathrm{r} ; \geq .20$ : mínimo recomendado; $\geq .50$ : moderado; $\geq .80$ : fuerte) y sus IC mediante un módulo MS Excel ${ }^{\circledR}$ creado para este caso (Caycho-Rodríguez, 2017). Finalmente, se examinó la confiabilidad de las puntuaciones de la SWLS mediante el cálculo del coeficiente de confiabilidad alfa de Cronbach (Cronbach, 1951) y según la recomendación de Nunnally y Bernstein (1994) valores mayores a .70 son adecuados.

\section{Resultados}

La escala se muestra sólida en su validez: la medida de adecuación muestral KMO es de .826 , lo cual indica que hay interdependencia entre los reactivos. La prueba de esfericidad de Bartlett muestra un aproximado de 971.62 y una $p<.001$. La prueba $z$ de Kolmogorov-Smirnov fue de 2.483, $\mathrm{p}=.001$, lo que indica que la muestra no se distribuye de manera normal. El análisis paralelo mostró una estructura monofactorial (un único autovalor $=1.197$ quedó por encima del punto de intersección). La varianza total explicada es de $64.17 \%$, con un solo factor. El alfa de Cronbach es de .850 mientras que el alfa de Cronbach con los elementos tipificados es 
de .858 , lo cual exhibe la precisión y la reproductibilidad de la prueba. Las cargas factoriales son elevadas (entre $\lambda=.576$ y .888 ; véase a continuación la Tabla 1), cumpliéndose el criterio de cargas factoriales superiores a $\lambda=.40$ (MacCallum, Widaman, Zhang, \& Hong, 1999; Velicer, \& Fava, 1998; Williams, Brown, \& Onsman, 2010). Por otra parte, el modelo de un solo factor presenta evidencia interna de validez convergente mediante el parámetro $\mathrm{AVE}=.58$ y de confiabilidad compuesta $\mathrm{FC}=.87$. Ambos índices AVE y FC obtuvieron valores mayores a los recomendados por Fornell y Larcker (1981).

Tabla 1.

\section{Cargas del análisis factorial exploratorio}

\begin{tabular}{ll}
\hline \multicolumn{1}{c}{ Cargas Factoriales de los ítems } & \\
\hline $\begin{array}{l}\text { 1. En la mayoría de los aspectos, mi } \\
\text { vida se acerca a mi ideal }\end{array}$ & .769 \\
$\begin{array}{l}\text { 2. Las condiciones de mi vida son } \\
\text { excelentes }\end{array}$ & .773 \\
$\begin{array}{l}\text { 3. Estoy completamente satisfecho(a) } \\
\text { con mi vida }\end{array}$ & .888 \\
$\begin{array}{l}\text { 4. Hasta ahora, he conseguido las } \\
\text { cosas más importantes que quiero en }\end{array}$ & .769 \\
$\begin{array}{l}\text { la vida } \\
\text { 5. Si pudiera vivir mi vida de nuevo, } \\
\text { no cambiaría nada }\end{array}$ & .576 \\
\hline
\end{tabular}

Como ya se había señalado, existe una interdependencia entre los ítems, así como correlaciones entre moderadas y altas inter-ítems. Esta interdependencia e interrelación en los ítems que componen la escala demuestran la solidez de la escala y su validez (Ver Tabla 2).

Como se puede observar en la Tabla 2, las correlaciones inter-ítems son altas y, como ya se señaló, muestran correlaciones entre moderadas y altas. Nuevamente el ítem número cinco es el que manifiesta la correlación más baja entre los ítems, sin embargo, cabe señalar que se encuentra por encima del .40.

Nuevamente los ítems muestran una clara interdependencia y una interrelación alta. En lo que respecta a las cargas factoriales, así como a la correlación si uno de los ítems es eliminado, nuevamente el ítem número cinco es el que representa un alfa de Cronbach más alta dado el caso de que se eliminara: .862 (Véase Tabla 3). También se puede observar que la correlación corregida ítem-total es más baja en el ítem número cinco: $r=.53$. Sin embargo, los cinco ítems de la SWLS no muestran correlaciones elevadas ( $\mathrm{r}$ $>$.90), por lo que se puede afirmar que no existe multicolinealidad (Tabachnick, Fidell, \& Osterlind, 2001) y de este modo garantizar

Tabla 2.

Matriz de correlaciones inter-item

\begin{tabular}{|c|c|c|c|c|c|}
\hline & 1 & 2 & 3 & 4 & 5 \\
\hline $\begin{array}{l}\text { 1. En la mayoría de los aspectos de mi } \\
\text { vida se acerca a mi ideal }\end{array}$ & 1 & & & & \\
\hline $\begin{array}{l}\text { 2. Las condiciones de mi vida son exce- } \\
\text { lentes }\end{array}$ & $.61 *$ & 1 & & & \\
\hline $\begin{array}{l}\text { 3. Estoy completamente satisfecho(a) con } \\
\text { mi vida }\end{array}$ & $.60 *$ & $.67 *$ & 1 & & \\
\hline $\begin{array}{l}\text { 4. Hasta ahora he conseguido las cosas } \\
\text { más importantes que quiero en la vida }\end{array}$ & $.58^{*}$ & $.49 *$ & $.69 *$ & 1 & \\
\hline $\begin{array}{l}\text { 5. Si pudiera vivir mi vida de nuevo, no } \\
\text { cambiaría nada. }\end{array}$ & $.41 *$ & $.43^{*}$ & $.54^{*}$ & $.43^{*}$ & 1 \\
\hline
\end{tabular}

$* p<.01$ 
que los ítems no son medidas redundantes de la satisfacción con la vida. Lo anterior brinda evidencia de validez interna discriminante (Merino-Soto, Domínguez-Lara, y Fernández -Arata, 2017).

En suma, de acuerdo a los hallazgos del análisis factorial exploratorio, el modelo de medición del constructo de Diener et al. (1985) se mantiene. Pero para una mayor seguridad al respecto, se informan a continuación los resultados sobre la escala mediante el análisis factorial confirmatorio.

\section{Análisis Factorial Confirmatorio}

Además de realizar el análisis factorial exploratorio, se sometió a la escala al análisis factorial confirmatorio para ver si la estructura factorial reportada por el primer tipo de análisis y la teorizada por los autores de la prueba se sostenía con la muestra de este estudio. Debido a que la $\mathrm{z}$ de Kolmogorov-Smirnov fue de 2.483, $\mathrm{p}=.001$, indicando una distribución no normal de las calificaciones en la escala, se usó el método de estimación por cuadrados mínimos libres de escala, en el análisis factorial confirmatorio, habiéndose encontrado una muy buena bondad de ajuste del modelo de medición, con los siguientes indicadores: $\mathrm{X}^{2} / \mathrm{gl}=5.291 / 5=1.0582 ; \mathrm{SRMR}=.029$; $\mathrm{RMR}=.065 ; \quad \mathrm{GFI}=.998 ; \quad \mathrm{AGFI}=.993$; $\mathrm{NFI}=.996$. Las $\mathrm{R}^{2}$ de los ítems de la escala alcanzaron valores muy aceptables, de 34\% (SV5), 56\% (SV1, SV2 y SV4) y de 78\% para el último (SV3) (Véase la Figura 1). Asimismo, las cargas factoriales $\left(\lambda^{\prime} s\right)$ de los ítems tuvieron un recorrido desde una mínima de .58 hasta una máxima de .88 (Véase la Figura 1). El coeficiente de consistencia interna

Tabla 3.

Análisis de la confiabilidad eliminando ítems

\begin{tabular}{|c|c|c|c|c|c|}
\hline & $\begin{array}{c}\text { Media de la } \\
\text { escala si se } \\
\text { elimina el } \\
\text { ítem } \\
\end{array}$ & $\begin{array}{c}\text { Varianza de } \\
\text { la escala si se } \\
\text { elimina el } \\
\text { ítem }\end{array}$ & $\begin{array}{l}\text { Correlación } \\
\text { ítem-total } \\
\text { corregida }\end{array}$ & $\begin{array}{l}\text { Correlación } \\
\text { múltiple al } \\
\text { cuadrado }\end{array}$ & $\begin{array}{c}\text { Alfa de } \\
\text { Cronbach si se } \\
\text { elimina el ítem }\end{array}$ \\
\hline $\begin{array}{l}\text { 1. En la mayoría de los } \\
\text { aspectos de mi vida se } \\
\text { acerca a mi ideal }\end{array}$ & 20.97 & 27.67 & .67 & .49 & .81 \\
\hline $\begin{array}{l}\text { 2. Las condiciones de } \mathrm{mi} \\
\text { vida son excelentes }\end{array}$ & 20.96 & 127.67 & .67 & .52 & .81 \\
\hline $\begin{array}{l}\text { 3. Estoy completamente } \\
\text { satisfecho(a) con mi vida }\end{array}$ & 20.75 & 24.83 & .78 & .64 & .78 \\
\hline $\begin{array}{l}\text { 4. Hasta ahora he conse- } \\
\text { guido las cosas más impor- } \\
\text { tantes que quiero en la vida }\end{array}$ & 20.95 & 26.22 & .67 & .52 & .81 \\
\hline $\begin{array}{l}\text { 5. Si pudiera vivir mi vida } \\
\text { de nuevo, no cambiaría } \\
\text { nada. }\end{array}$ & 21.05 & 25.75 & .53 & .30 & .86 \\
\hline
\end{tabular}




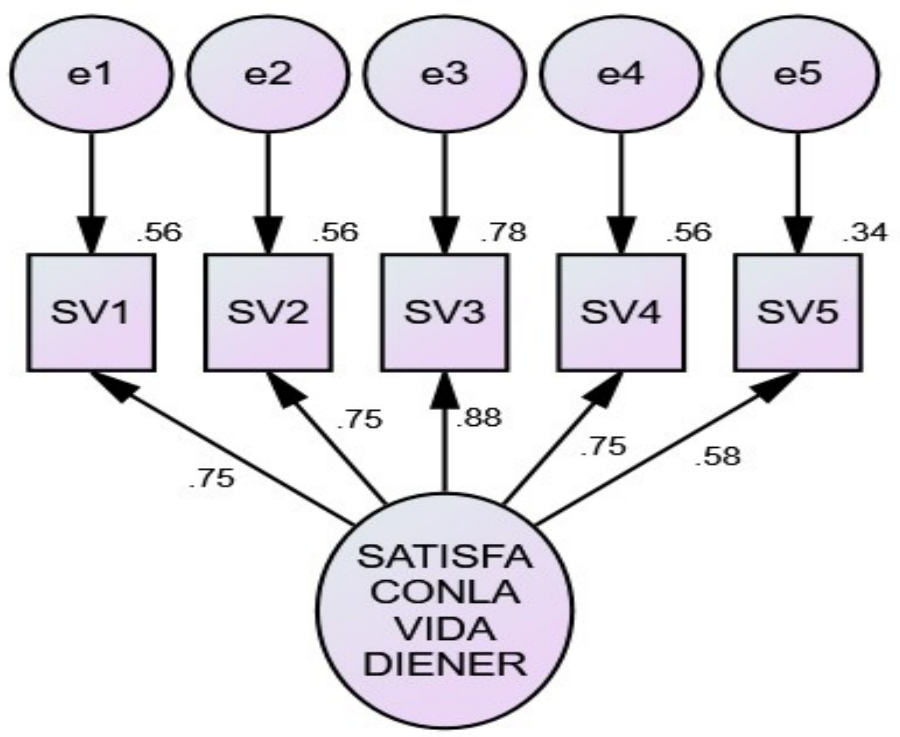

Figura 1. Modelo estandarizado de la prueba Satisfacción con la Vida, usando cuadrados mínimos libres de escala como método de estimación.

alfa de Cronbach fue de .85 , con un mínimo de .826 y máximo de .871 , en un intervalo de confianza del 95\%.

\section{Validez convergente}

Se evaluó la correlación entre la SWLS (Diener et al., 1985) y la escala de Calidad de Vida (Palomar, 1995) como indicador de la validez convergente. El coeficiente de correlación de Pearson entre la SWLS y la escala de Calidad de Vida fue de $\mathrm{r}=.395 ; \mathrm{p}<.01$; IC95\%: .293, .486. Lo anterior muestra que la correlación tiene la dirección esperada y es estadísticamente significativa. Respecto al TE la correlación entre la SWLS y la escala de Calidad de Vida presenta un TE mínimo deseado $(r \geq .20)$.

\section{Discusión}

Con respecto a la primera hipótesis de este estudio, la Escala de Satisfacción con la Vida de Diener et al. (1985) demuestra tener consistencia interna, en este caso con población juvenil de edad escolar de bachillerato, con un valor muy cercano al obtenido por Atienza et al. (2000) en una muestra de adolescentes, así como también los datos obtenidos en un estudio transcultural con países europeos de España y Portugal (Atienza et al., 2016). Dichos valores son similares a los obtenidos en América Latina, específicamente en Chile, Colombia y en México (Cárdenas et al., 2012; Díaz et al., 2015; Dimitrova \& Domínguez, 2015; Padrós et al., 2015). Los hallazgos se unen a los reportados por estudios que se han efectuado desde la propuesta original de Diener et al. (1985). Se apoya el modelo conceptual y empírico de una escala con una estructura monofactorial (Atienza et al., 2016; Cárdenas et al., 2012; Díaz et al., 2015; Diener et al., 1985; Diener, Horowitz et al., 1985; Dimitrova \& Domínguez, 2015; Magalhães \& Calheiros, 2015; Pavot \& Diener, 1993).

En relación a la segunda hipótesis, la escala de satisfacción con la vida muestra una validez convergente con la escala de calidad de vida, lo que da apoyo a la argumentación de ser componentes del bienestar subjetivo, 
sin embargo, ambas poseen sus características que las diferencian.

Un aspecto importante para la discusión en este estudio es si las conceptualizaciones de los fenómenos psicológicos y sus respectivas operacionalizaciones a través de diferentes instrumentos, responden a cuestiones de la naturaleza universal o cultural de la personalidad de los individuos; en este caso concreto de la escala de satisfacción con la vida, es abrumadora la evidencia hacia la primera de las dos interpretaciones: la universalista. Hasta este momento, parece que la teoría de Diener et al. (1985) sobre la satisfacción con la vida, y la escala que propusieron, resulta válida y confiable, en todas las culturas en las que se ha investigado. Esto no significa que lo será en todas las que faltan por explorar, pero tiene a favor una gran probabilidad. Por tanto, aparentemente, la pregunta no es si responde a la naturaleza universal de los seres humanos o no, sino por qué. Si se analizan a qué se refieren las frases de las cuales consta la escala, hay afirmaciones sobre lo más valioso que tiene uno, lo cual es su propia vida, realizando juicios que se plantean básicamente en el pasado: "Hasta el momento, he conseguido las cosas importantes que quiero en la vida" y "Si pudiera volver a vivir mi vida, no cambiaría casi nada" y también el presente: "Estoy satisfecho con mi vida", "Las condiciones de mi vida son excelentes" y "Mi vida, en casi todo, responde a mis aspiraciones". Estas dos características de la escala: 1) referirse a la propia vida, aquello con lo cual cargamos (para bien o para mal) todos los días o por antonomasia, lo que somos, supuestamente conocemos más y sin duda amamos más que a nada en esta tierra y 2) tratar sobre cómo nos ha ido (dimensión del pasado) y nos está yendo (dimensión presente) a nosotros (somos nuestra propia vida), implican com- partir algo muy elemental y común a todos los que pertenecemos a la especie Homo Sapiens, independientemente de cualquier aspecto social o cultural. Por tanto, ello ayudaría a entender la asombrosa "universalidad" de la escala, traducida en validez de constructo y muy buenos coeficientes de consistencia alfa de Cronbach.

Por otra parte, en cierto sentido, esta teorización y el instrumento respectivo de la satisfacción con la vida de Diener et al. (1985) coinciden con el razonamiento y su operacionalización del constructo optimismo de García-Cadena, Téllez, Ramírez, Ramírez y Pérez (2016) porque tanto la satisfacción con la vida como el optimismo se refieren a la vida dentro de las dimensiones pasada y presente, y aun cuando sí se logró evidencia de validez convergente de la escala de GarcíaCadena et al. (2016) con la escala de optimismo de Scheier, Carver y Bridges (1994), el grado de traslape conceptual entre la de satisfacción con la vida de Diener et al. (1985) y la de García-Cadena et al. (2016) merece una investigación por su propio derecho. Se propone que en lo posible se usen ambas escalas con muestras mexicanas similares, de diferente edad y de distintos estratos escolares, para encontrar que tanto se traslapan conceptualmente.

A manera de conclusión, se puede decir que la escala de satisfacción con la vida posee propiedades psicométricas muy parecidas a las obtenidas tanto dentro de México, aun y cuando las muestras han sido de grupos donde la etapa del ciclo vital son disimiles, y como hallazgo de interés, confirmando una estructura monofactorial en las diferentes culturas donde se ha estudiado. La evidencia nos revela un instrumento adecuado para medir uno de los componentes del bienestar subjetivo, el cual resulta de gran interés para la etapa 
de la adolescencia donde se ha encontrado que hay una tendencia a presentarse situaciones de riesgos (drogadicción, conductas violentas, embarazos precoces, etc.), las que han sido asociadas con una baja satisfacción con la vida. Además, constituye una escala que requiere de invertir poco tiempo para su aplicación, lo cual facilita su uso.

Una de las limitaciones de la presente investigación se refiere a la muestra en la que se aplicó la escala, ya que siendo de adolescentes escolarizados, no se pueden generalizar los resultados a todos los adolescentes, y menos a los que no se encuentran en sistemas educativos formales. Esto abre la oportunidad para seguir indagando en una población de adolescentes, donde la variable de la educación no esté limitada por el nivel de bachillerato.

También queda abierta la línea de investigación donde se puede ver el valor predictivo del bienestar subjetivo, en especial los componentes de la satisfacción con la vida, calidad de vida, el optimismo en situaciones problemáticas a nivel fisico, cognitivo y emocional en la adolescencia.

\section{Referencias}

Abu-Bakar, A. (2012). Health-related quality of life among youth: Evaluating measurement model fit. International Journal of Social Science and Humanity, 2(3), 282-284. doi:10.7763/ JSSH.2012.V2.110

Alarcón, R. (2006). Desarrollo de una escala factorial para medir a felicidad. Revista Interamericana de Psicología, 40(1), 99-106.

Atienza, G. F. L., Balaguer, S. I., Corte-Real, N., \& Fonseca, A. M. (2016). Factorial invaraince of the Satisfaction with Life Scale in adolescents from Spain and Portugal. Psicothema, 28(3), 353-358. doi:10.7334/psicothema2016.1

Atienza, G. F. L., Pons, D., Balaguer, S. I., y GarcíaMerita, M. (2000). Propiedades psicométricas de la escala de satisfacción con la vida adolescente. Psicothema, 12(2), 314-319.
Bollen, K.A. (1998). Structural equation models. En P. Armitage \& T. Colton (Eds.). Encyclopedia of Biostatistics (pp. 4363-4372). Sussex, UK: Wiley.

Cabañero, M. M. J. Richart, M. M., Cabrero, G. M.I., Orts, C. M. I., Reig, F. A., y Tosal, H. B. (2004). Fiabilidad y validez de la Escala de Satisfacción con la Vida de Diener en una muestra de mujeres embarazadas y puérperas. Psicothe$m a, 16(3), 448-455$.

Cárdenas C., M., Barrientos D., J., Bilbao R., Á., Páez R., D., Gómez O., D., y Asún S., D. (2012). Estructura factorial de la escala de satisfacción con la vida en una muestra de estudiantes universitarios chilenos. Revista Mexicana de Psicología, 29(2), 157-164.

Castro, S. A. (2009). El bienestar psicológico cuatro décadas de progreso. Revista Interunivesritaria de formación del profesorado, 66(23, 3), 43-72

Caycho-Rodríguez, T. (2017). Tamaño del efecto e intervalos de confianza para correlaciones: aportes a Montes Hidalgo y Tomás-Sábado. Enfermería Clínica, 27(5), 331-2. doi:10.1016/ j.enfcli.2017.07.001

Courtney, M. (2013). Determining the Number of Factors to Retain in EFA: Using the SPSS R-Menu v2.0 to Make More Judicious Estimations. Practical Assessment, Research \& Evaluation, 18(8), 1-14. Recuperado de https://pareonline.net/ getvn.asp? $\mathrm{v}=18 \% 26 \mathrm{n}=8$

Cronbach, L.J. (1951). Coefficient alpha and the internal structure of test. Psychometrika, 16(3), 297334.

Díaz, D., Stavraki, M., Blanco, A., \& Gandarillas, B. (2015). The eudaimonic component of satisfaction with life and psychological well-being in Spanish cultures. Psicothema, 27(3), 247-253. doi:10.7334/psicothema2015.5

Diener, E. (1984). Subjective well-being. Psychological Bulletin, 95(3), 542-575. doi:10.1037/00332909.95.3.542

Diener, E., Emmons, R., Larsen, R. J., \& Griffin, S. (1985). The Satisfaction with Life Scale. Journal of Personality Assessment, 49 (1), 71-75. doi:10.1207/s15327752jpa4901_13

Diener, E., Horowitz, J., \& Emmons, R. A. (1985). Happiness of the very wealthy. Social Indicators Research, 16 (3), 263-274. doi:10.1007/ BF00415126

Diener, E., Oishi, S., \& Lucas, R. E. (2003). Personality, culture, and subjective well-being: Emotional and cognitive evaluations of life. The Annual 
Review of Psichology, 54, 403- 425. doi:10.1146/annurev.psych.54.101601.145056

Dimitrova, R., \& Domínguez, E. A.E. (2015). Measurement invariance of the Satisfaction with Life Scale in Argentina, Mexico and Nicaragua. Social Inquiry into Well-Being, 1(1), 32-39. doi:10.13165/SIIW-15-1-1-04.

Escobar, E. M., Blanca, M.J., Fernández-Baena, F. J., y Trianes, T. M. V. (2011). Adaptación española de la escala de manifestaciones de estrés del Student Stress Inventary (SSI-SM). Psicothema, 23(3), 475-485.

Fischer, R., \& Boer, D. (2011). What is more important for national well-being: money or autonomy? A meta-analysis of well-being, burnout, and anxiety across 63 societies. Journal of Personality and Social Psychology, 101(1), 164184. doi:10.1037/a0023663.supp

Fornell, C., \& Larcker, D. F. (1981). Evaluating structural equation models with unobservable variables and measurement error. Journal of Marketing Research, 18(1), 39- 50. doi: $10.2307 / 3151312$.

Fujikoshi. Y. (2000). Transformations with improved chi-squared approximations. Journal of Multivariate Analysis, 72(2), 249-63. doi:10.1006/ jmva.1999.1854.

García-Cadena, C.H., Téllez, A., Ramírez, G., Ramírez, E., \& Pérez, E.J. (2016). Toward a global conceptualization and measurement in positive psychology. En R. Bowers (Ed.), Psychological well-being: Cultural influences, measurement strategies and health implications (pp. 21-42). Nueva York: Nova Science Publishers, Inc.

Garrido, M.M. R., Fernández, B. M. A., Villalba, R. E. B., Pérez, M. P. J., y Fernández, B. M. (2010). Evidencias de validez de la adaptación al español de la Escala de Satisfacción con la Vida en una muestra de estudiantes universitarios. Metodología de Encuestas, 12(1), 45-62.

Gwozd W., \& Sousa-Poza, A. (2009) Ageing, health and life satisfaction of the oldest old: An analysis for Germany. IZA Discussion Paper No. 4053, 1-37.

Hancock, G.R., \& Mueller, R.D. (2006). Structural Equation Modeling, A Second Course. Greenwich, Connecticut: IAP.

Herian, M. N., Tay, L., Hamm, J. A., \& Diener, E. (2014). Social capital, ideology, and health in the United States. Social Science \& Medicine, 105, 30-37. doi:0.1016/j.socscimed.2014.01.003

Hu, L., \& Bentler, P. (1999). Cutoff criteria for fit in- dexes in covariance structure analysis: Conventional criteria versus new alternatives. Structural Equation Modeling: A Multidisciplinary Journal, 6(1), 1-55. doi:0.1080/10705519909540118

Instituto Nacional de Estadística y Geografía [INEGI] (2015). Encuesta intercensal. Principales resultados. Recuperado de http:// www.beta.inegi.org.mx/contenidos/proyectos/ enchogares/especiales/intercensal/2015/doc/ eic_2015_presentacion.pdf

Jahoda, M. (1958). Current concepts of positive mental health. Nueva York: Basic Books.

Keyes, C. (1998). Social well-being. Social Psychology Quarterly, 61(2), 121-140. doi: $10.2307 / 2787065$

Laca, F. A., Verdugo, J. C., y Guzmán, J. (2005). Satisfacción con la vida de algunos colectivos mexicanos: una discusión sobre la psicología del bienestar subjetivo. Enseñanza e Investigación en Psicología, 10(2), 325-336.

Lee, M., Lee, H. I., \& Rojewski, J. W. (Noviembre 2012). The role of contextual and intrapersonal factors in students' school satisfaction in Korean vocational high schools. Póster presentado en Conference of the Association for Career and Technical Education Research. Atlanta, GA.

Lozano, V. A. (2014). Teoría de teorías sobre la adolescencia. Última década, 40, 11-36.

Lloret-Segura, S., Ferreres-Taver, A., HernándezBaeza, A., \& Tomás-Marco, I. (2014). El análisis factorial exploratorio de los ítems: una guía práctica, revisada y actualizada. Anales de Psicología, 30(3), 1151-1169. doi:10.6018/ analesps.30.3.199361

Lucas, R. E., Diener, E., \& Suh, E. (1996). Discriminant validity of well-being measures. Journal of Personality and Social Psychology, 71(3), 616628. doi:10.1037//0022-3514.71.3.616

MacCallum, R. C., Widaman, K. F., Zhang, S. \& Hong, S. (1999). Sample size in factor analysis. Psychological Methods, 4(1), 84-99.

Magalhães, E., \& Calheiros, M. M. (2015). Psychometric properties of the Portuguese version of place attachment scale for youth in residential care. Psicothema, 27(1), 65-73. doi:10.7334/ psicothema2014.40

Martínez G., M. D., González -Arratia, L. N., van Barneveld, H. O., y Domínguez E., A. del C. (2012). Satisfacción con la vida asociada al apoyo familiar en la perimenopausia y posmenopausia. Salud Mental, 35(2), 91-98. doi:10.5294/aqui.2012.12.3.8 


\section{Revista de Psicología y Ciencias del comportamiento de la Unidad Académica de Ciencias Jurídicas y Sociales \\ Vol. 9. Núm. 2 (julio-diciembre 2018) \\ Martell, J.; García-Cadena, C.; Daniel-González, L.; Sánchez, M. y Mendoza, A.}

Martínez U. P. (2006). Prespectiva future del Perú y nivel de satisfacción con él. Liberabit, 12, 113121.

Merino-Soto, C., Domínguez-Lara, S., y FernándezArata, M. (2017). Validación inicial de una Escala Breve de Satisfacción con los Estudios en estudiantes universitarios de Lima. Educación Médica, 18(1), 74-77. doi:10.1016/ j.edumed.2016.06.016.

Montero, I., y León, O. G. (2005). Sistema de clasificación del método en los informes de investigación en Psicología. International Journal of Clinical and Health Psychology, 5(1), 115-127.

Myers, D., \& Diener, E. (1995). Who is happy? Psychological Science, 6(1), 10-17. doi:10.1111/ j.1467-9280.1995.tb00298.x

Nunnally, J. C., \& Bernstein, I. H. (1994). Psychometric Theory. New York, NY: McGraw-Hill.

Organización de las Naciones Unidas (ONU 2010). Programa de Acción Mundial para los Jóvenes (PAMJ). Recuperdo de: https://www.un.org/esa/ socdev/documents/youth/publications/ wpay2010SP.pdf

Padrós, F., Gutiérrez, C. Y., y Medina, M. A. (2015). Propiedades psicométricas de la escala de satisfacción con la vida (SWLS) de Diener en población de Michoacán (México). Avances en Psicología Latinoamericana, 33(2), 223-232. DOI: 10.12804/apl33.02.2015.04

Palomar, J. (1995). Diseño de un Instrumento de Medición de Calidad de Vida (Tesis de maestría). Universidad Nacional Autónoma de México, Ciudad de México, México.

Palomar, J. (1999). Relación entre el funcionamiento familiar y la calidad de vida en familias con un miembro alcoholico. Salud Mental, 22(6), 1321.

Pavot, W., \& Diener. E., (1993). The affective and cognitive contest of self-reports measures of subjective well-being. Social Indicators Research, 28(1), 1-20. doi:10.1007/BF01086714

Papalia, D. E., Wendkos, O., S, y Duskin, F. R. (2009). Adolescencia. Psicología del desarrollo (pp. 458 - 545). México: McGraw-Hill.

Pedhazur, E. J., \& Pedhazur Schmelkin, L. (1991). Measurement, design, and analysis. An integrated approach. Hillsdale: Lawrence Erlbaum Associates Inc.

Proctor, C. Linley, P. A., \& Maltby, J. (2009). Youth life satisfaction measures: A review. The Journal of Positive Psychology, 4(2), 128-144. doi:10.1080/17439760802650816
Rodríguez, Y. R., y Quiñones, B. A. (2012). El bienestar psicológico en el proceso de ayuda con estudiantes universitarios. Revista Griot, 5 (1), 7-17.

Ryff, C., \& Singer, B. (1998). The contours of positive human health. Psychological Inquiry, 9(1), 128. doi:10.1207/s15327965pli0901_1

Sánchez, E. M. J., García, M. J.M., Valverde, R. M., y Pérez, A. M. (2014). Enfermedad crónica: satisfacción vital y estilos de personalidad adaptativo. Clínica y Salud, 25(2), 85-93. doi:10.1016/ j.clysa.2014.06.007

Scheier, M.F., Carver, C.S., \& Bridges, M.W. (1994). Distinguishing optimism from neuroticism (and trait anxiety, self-mastery, and self-esteem): A reevaluation of the Life Orientation Test. Journal of Personality and Social Psychology, 67 (6), 1063-1078. doi:10.1037/00223514.67.6.1063

Sociedad Mexicana de Psicología (2007, 4a. edición). Código Ético del Psicólogo. México: Trillas.

Sun, R., \& Shek, D. (2012). Positive youth development, life satisfaction and problem behaviour among Chinese adolescents in Hong Kong: A replication. Social Indicators Research, 105, 541-559. doi:10.1007/s11205-011-9786-9

Sun, S., Chen, J., Johannesson, M., Kind, P., \& Burström, K. (2015). Subjective well-Being and its association with subjective health status, age, sex, region, and socio-economic characteristics in a Chinese population study. Journal of Happiness Studies, 17(2), 833-873. doi:10.1007/ s10902-014-9611-7

Tabachnick, B. G., Fidell, L. S., \& Osterlind, S. J. (2001). Using Multivariate Statistics (4th ed.). Boston: Allyn \& Bacon.

Tov, W., \& Diener, E. (2007). Culture and subjective well-being. En S. Kitayama \& D. Cohen (eds). Handbook of cultural psychology (pp. 691713). New York: Guilford.

Vázquez, C., Duque, A., \& Hervás, G. (2013). Satisfaction with Life Scale in a Representative Sample of Spanish Adults: Validation and Normative Data. The Spanish Journal of Psychology, 16, e82. doi:10.1017/sjp.2013.82

Veenhoven, R. (2008). Healthy happiness: Effects of happiness on physical health and the consequences for preventive health care. Journal of Happiness Studies, 9(3), 449-469. doi:10.1007/ s10902-006-9042-1

Velicer, W. F. \& Fava, J. L. (1998). Effects of variable and subject sampling on factor pattern recovery. 
Psychological Methods, 3(2), 231-251.doi: 10.1037/1082-989X.3.2.231

Villa T., I. G., (2015). Estilo de pensamiento rumiativo y bienestar subjetivo en escolares de quinto año de secundaria. (Tesis de Licenciatura). Pontificia Universidad Católica del Perú, Lima, Perú. Recuperado en: http://tesis.pucp.edu.pe/ repositorio/handle/123456789/6528

Werner, E., \& Smith, R. (1982). Vulnerable but invincible: A longitudinal study of resilient children and youth. Nueva York: McGraw Hill.

Williams, B., Brown, T., \& Onsman, A. (2010). Exploratory factor analysis: A five-step guide for novices. Australasian Journal of Paramedicine, 8(3), 1-13.

Wilson, W. (1967). Correlates of a wowed happiness. Psychological Bulletin, 67(4), 294-306.

Yiengprugsawan, V., Somboonsook, B., Sam-ang, S., \& Sleigh, A. C. (2012). Happiness, mental health, and socio-demographic associations among a national cohort of Thai adults. Journal of Happiness Studies, 13, 1019-1029. doi:10.1007/s10902-011-9304-4

You, S., Furlong, M. J., Felix, E., Sharkey, J. D., Tanigawa, D., \& Green, J. G. (2008). Relations among school connectedness, hope, life satisfaction, and bully victimization. Psychology in the Schools, 45(5), 446-460. doi:10.1002/ pits. 20308

Zagórski, K., Kelley, J., \& Evans, M. (2010). Economic development and happiness: Evidence from 32 nations. Polish Sociological Review, 169(1), 3-19. 